\title{
Targeting insulin-producing beta cells for regenerative therapy
}

\author{
Adriana Migliorini $^{1,3}$ - Sara S. Roscioni ${ }^{1,3} \cdot$ Heiko Lickert $^{1,2,3}$
}

Received: 23 November 2015 / Accepted: 13 January 2016/Published online: 13 July 2016

(C) Springer-Verlag Berlin Heidelberg 2016

\begin{abstract}
Pancreatic beta cells differ in terms of glucose responsiveness, insulin secretion and proliferative capacity; however, the molecular pathways that regulate this cellular heterogeneity are unknown. We have identified the Wnt-planar cell polarity (PCP) effector Flattop (FLTP) as a biomarker that identifies mature beta cells in the islets of Langerhans. Interestingly, three-dimensional architecture and Wnt-PCP ligands are sufficient to trigger mouse and human beta cell maturation. These results highlight the fact that novel biomarkers shed light on the long-standing mystery of beta cell heterogeneity and identify the Wnt-PCP pathway as triggering beta cell maturation. Understanding heterogeneity in the islets of Langerhans might allow targeting of beta cell subpopulations for regenerative therapy and provide building principles for stem cell-derived islets. This review summarises a presentation given at the 'Can we make a better beta cell?' symposium at the 2015 annual meeting of the EASD. It is accompanied by two other reviews on topics from this symposium (by Amin Ardestani and Kathrin Maedler, DOI: 10.1007/s00125-016-3892-9, and by Harry Heimberg and colleagues, DOI: 10.1007/s00125-0163879-6) and a commentary by the Session Chair, Shanta Persaud (DOI: 10.1007/s00125-016-3870-2).
\end{abstract}

Heiko Lickert

heiko.lickert@helmholtz-muenchen.de

1 Institute of Diabetes and Regeneration Research, Helmholtz Zentrum München, Ingolstädter Landstr. 1, D-85764 Neuherberg, Germany

2 Institute of Stem Cell Research, Helmholtz Zentrum München, Munich, Germany

3 German Center for Diabetes Research (DZD), Neuherberg, Germany
Keywords Beta cell heterogeneity $\cdot$ Cell-replacement therapy $\cdot$ Dedifferentiation $\cdot$ Islet architecture $\cdot$ Maturation . Plasticity $\cdot$ Regenerative therapy $\cdot$ Wnt signalling
Abbreviations
3D Three-dimensional
FLTP Flattop
GSIS Glucose-stimulated insulin secretion
PCP Planar cell polarity

Loss of insulin-producing beta cell mass and function underlies the pathogenesis of both type 1 and type 2 diabetes mellitus. Ideally, the best treatment would stop the progression of the disease by regenerating functional cells from dysfunctional beta cell mass or by replacing the beta cells with functional new ones. Lost beta cell mass has been successfully replenished by islet transplantation according to the Edmonton protocol [1]. This treatment normalises blood glucose levels, reduces secondary complications and improves quality of life. Besides beta cell replacement, increasing evidence indicates that in vivo beta cell regeneration is a promising treatment option for both types of diabetes. Importantly, the Joslin Medalist Study demonstrated that even after 50 years of insulin-dependent autoimmune diabetes, there is residual beta cell turnover and insulin is secreted into the bloodstream [2]. Strikingly, patients were protected from secondary complications, suggesting that even low levels of insulin can buffer glucose excursion and protect against long-term organ damage [3]. Thus, triggering endogenous mechanisms of beta cell regeneration might be a suitable alternative for diabetes therapy. 
Is beta cell regeneration possible in diabetic patients?

If such an ambitious goal is to be reached, it is crucial to know how beta cell regeneration can be achieved in diabetic patients. During development and maturation and from birth to death, beta cells can alter their identity and their functionality. Over the last 30 years, beta cells have been discovered to be a heterogeneous population of cells in terms of glucose responsiveness, metabolic flux, insulin secretion, topography and proliferative capacity [4-6]. Heterogeneity may not only be caused by pathological dedifferentiation of beta cells [7, 8], but may also stem from the different origins of beta cells. Dor et al postulated that self-replication of pre-existing beta cells is the major mode regulating beta cell replenishment in adult islets [9]. Others have suggested that self-renewing and multipotent beta cell progenitors co-exist in mouse and human islets [10]. Moreover, facultative stem cells in the duct, or transdifferentiation of other islet cell types, might represent alternative sources of heterogeneous beta cell pools [11-13]. Accordingly, several independent studies in recent years have demonstrated that manipulation of a single gene or beta cell depletion is sufficient to induce islet cell plasticity and drive the transdifferentiation of alpha or delta cells towards the beta cell lineage, as well as to enhance the formation of new beta cells from ductal facultative stem cells $[11,14-16]$. This obvious islet cell plasticity might influence the gene expression profile and function of the beta-like cells. Alternatively, the three-dimensional (3D) islet architecture and interaction with neighbouring cell types that determine the beta cell niche might affect the function and maturation status depending on topography and the particular next-door neighbour. Identifying marker genes and underlying principles of beta cell heterogeneity might therefore enable the targeting of specific beta cell subpopulations for regenerative strategies in diabetic patients.

\section{Can we target beta cell heterogeneity for regenerative therapy?}

Towards this goal we have recently identified a novel biomarker named Flattop (Fltp), which is heterogeneously expressed among pancreatic endocrine cells (Fig. 1). Fltp was originally discovered as a functionally non-annotated gene $(1700009 P 17 R i k)$ in a screen to identify forkhead box protein A2 (FOXA2) target genes involved in polarity establishment $[17,18]$ and is expressed in regions that depend on non-canonical Wnt-planar cell polarity (PCP) signalling $[19,20]$. The conserved canonical and non-canonical Wnt pathways have been shown to be critical for developmental patterning, organ morphogenesis, establishment of tissue polarity and cell differentiation [21-24]. The Wnt-PCP pathway regulates the orientation of cells and organelles within the plane of tissue and thus critically determines the function of cells in an organ $[22,25]$. Two recent independent studies have demonstrated that Wnt-PCP signalling plays a pivotal role first during endoderm-derived organ formation, governing liver vs pancreas specification, and later in the developing pancreas, regulating beta cell differentiation $[26,27]$. Fltp is a novel Wnt-PCP effector and reporter gene that is transcriptionally activated in mono- and multiciliated tissues while PCP is being acquired. Fltp functions downstream of the core Wnt-PCP molecules as an effector by regulating the actin and microtubule cytoskeleton. Interestingly, lineage-tracing analysis revealed that Fltp is expressed in definitive endoderm-derived organs, like the lung and endocrine cells of the developing pancreas [20]. Based on the recent observation that the Wnt-PCP pathway, tissue organisation and neighbouring cell type interactions are important for the generation of the proper amount of beta cells [27], we investigated the expression and function of our novel Wnt-PCP effector molecule FLTP in the islets of Langerhans.

During islet neogenesis in the mouse, immature/naive beta cells are still organised in solitary or cord-like structures and acquire the typical spherical 3D islet architecture in the first 2 weeks after birth. These naive beta cells proliferate extensively, secrete insulin in response to low glucose levels and do not reach maturation until the islets are formed [28]. Indeed, analysis of pancreases from our Fltp reporter mice revealed increasing reporter activity in beta cells of compacted islets, whereas reporter activity was undetectable in cord-like structures at embryonic day (E) 18.5. This suggests that while the islet is in the process of acquiring its 3D structure, the WntPCP pathway, monitored by Fltp expression, is activated and regulates islet neogenesis and maturation.

In adult rodent islets, most but not all beta cells are organised around vessels in rosette-like structures to secrete insulin into the bloodstream [29]. It is uncertain whether this difference in tissue distribution relates to the previously described heterogeneity of beta cells in terms of maturation, granularity, size, insulin secretion capacity and glucose responsiveness [4, 30-32]. Interestingly, during beta cell maturation and islet formation, Fltp expression rapidly increased, with the result that $80 \%$ of adult beta cells were Fltp reporter ${ }^{+}$, which might require Wnt-PCP function to wrap around blood vessels in rosette-like structures and be efficiently coupled for insulin secretion and glucose responsiveness. Furthermore, Fltp reporter beta cells show an up to fourfold increased proliferation rate during pregnancy and postnatal beta cell expansion [33]. This suggests that FLTP is a novel biomarker that subdivides beta cells into proliferative and mature cell types (Fig. 1).

Genome-wide mRNA expression profiling of flow cytometry-sorted Fltp reporter ${ }^{-}$and Fltp reporter ${ }^{+}$endocrine subpopulations revealed that the Fltp reporter population associates with gene ontology (GO) terms such as GPCR, 
Fig. 1 Beta cell heterogeneity in the islets of Langerhans. FLTP is a novel biomarker that is heterogeneously expressed among beta cells. Wnt-PCP and 3D architecture trigger Fltp expression and beta cell maturation. Fltp reporter ${ }^{+}$beta cells are more metabolically active and undergo hypertrophy upon diet-induced obesity, whereas Fltp reporter beta cells show an increased proliferation rate in response to metabolic demand. INS ${ }^{+}$, insulin-positive; MafA, v-maf avian musculoaponeurotic fibrosarcoma oncogene homologue A; NKX6.1 $1^{+}$, NK6 homeobox 1-positive

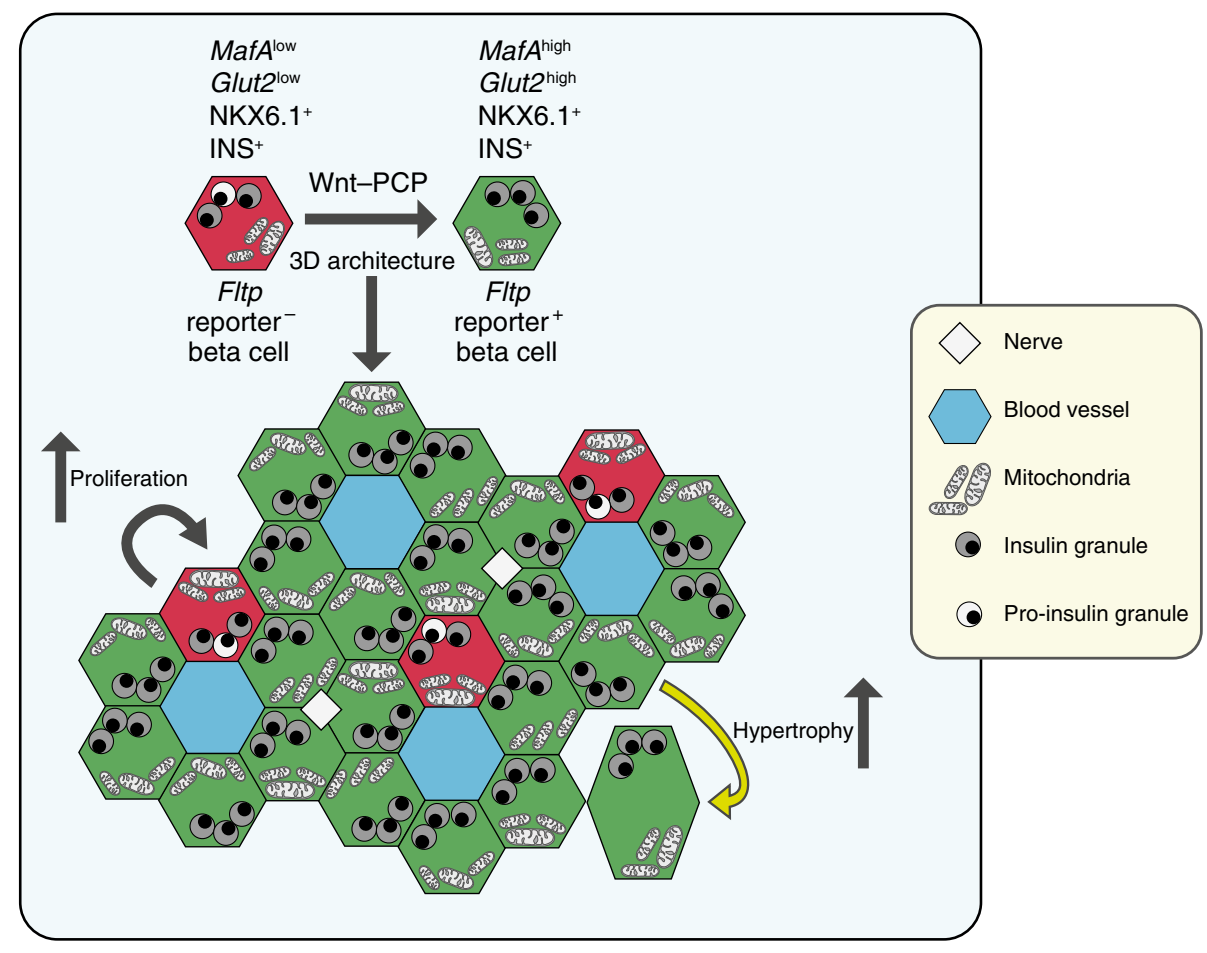

MAPK and Wnt signalling, whereas the Fltp reporter ${ }^{+}$ population associates with $\mathrm{GO}$ terms such as insulin secretion, glucose metabolism, mitochondria organisation, oxidative stress response and cellular respiration. This suggested that we had identified a biomarker for beta cell subpopulations with distinct molecular, physiological and ultrastructural features, and was confirmed experimentally. The differential expression of receptors and signalling pathway components further suggested that these beta cell subpopulations might react differentially to environmental changes. To explore this idea, we investigated the in vivo dynamics of these two populations by genetic lineage tracing under conditions of physiological and pathological insulin resistance. Flow cytometry analysis of the lineage-traced FLTP subpopulations upon physiological insulin resistance revealed that FLTP lineage ${ }^{-}$beta cells are responsible for beta cell expansion, which is likely to be followed by subsequent differentiation into FLTP lineage ${ }^{+}$beta cells [33].

Longitudinal live-cell imaging of transplanted islets in the anterior chamber of the eye of the host mouse [34] showed increases in cell volume and the number of FLTP lineage ${ }^{-}$ cells, and these occurred in parallel with islet engraftment on the iris, during which islets become vascularised and innervated and gain functionality. On the other hand, 8 weeks of diet-induced obesity and induction of pathological insulin resistance produced a steady growth of the islet volume accompanied by a significant increase in cross-sectional area of the FLTP lineage ${ }^{+}$endocrine cell population, suggesting that this beta cell subpopulation accounts for pathological islet cell hypertrophy. These results are consistent with the idea that
FLTP lineage $^{+}$cells have increased metabolic activity. Finally, we have shown that Fltp gene function is not necessary for beta cell development or maturation, but is required for proper glucose-stimulated insulin secretion (GSIS), perhaps by regulating the actin and microtubule cytoskeleton [35]. Interestingly, in 2D and 3D culture we demonstrated that 3D architecture and the Wnt-PCP pathway are sufficient to trigger mouse and human beta cell maturation and improve GSIS. Overall, these findings confirm that the Wnt-PCP effector and reporter gene Fltp subdivides proliferative and mature beta cells. These beta cell pools react differently to environmental changes. Importantly, the differential expression of receptors and signalling molecules suggests that beta cell subpopulations can be targeted to enhance proliferation or maturation for regenerative therapy.

\section{Beta cell heterogeneity: implications for therapy}

Although the understanding of beta cell biology has enormously increased since the discovery of the islets of Langerhans, there is much more to unveil before cell replacement and regeneration therapy can enter the clinic. Markers of functional beta cell heterogeneity will increase the understanding of the underlying principles of beta cell plasticity, dedifferentiation and maturation. For example, these markers could offer insights into the mechanisms that orchestrate beta cell maturation and could therefore be used for drug screens aiming to trigger the missing final step of maturation of in vitro differentiated beta-like cells from stem cells. 
Alternatively, these markers may improve the understanding of the islet niche and 3D architecture to create functional islets in a tissue engineering approach. In this regard, our findings support the concept that non-canonical Wnt-PCP-mediated tissue polarisation and compaction of islets are responsible for endocrine cell maturation and function. Biomarkers such as FLTP will help to unravel pathogenic mechanisms of diabetes and identify beta cell subpopulations in situ under conditions known to affect beta cell mass and function. Collectively, this could open a new era in the treatment of diabetes, paving the way for regenerative therapy for diabetic patients.

Acknowledgements We are grateful to E. Bader and M. Gegg (Institute of Diabetes and Regeneration Research, Helmholtz Zentrum München, Neuherberg, Germany) for comments and discussions.

Funding AM is funded by a Helmholtz post-doctoral fellowship. Work in the authors' laboratories is supported by the Helmholtz Alliance ICEMED - Imaging and Curing Environmental Metabolic Diseases, through the Initiative and Networking Fund of the Helmholtz Association and the German Center for Diabetes Research (DZD e.V.).

Duality of interest $\mathrm{HL}$ and AM are inventors of a European Patent for FLTP as a marker for beta cell maturation (EP14161818 and PCT/ EP2015/056664). SSR has no duality of interest associated with this manuscript.

Contribution statement AM, SSR and HL conceived, wrote and revised the manuscript. All authors approved the final version of the manuscript.

\section{References}

1. Shapiro AM, Lakey JR, Ryan EA et al (2000) Islet transplantation in seven patients with type 1 diabetes mellitus using a glucocorticoid-free immunosuppressive regimen. N Engl J Med 343:230-238

2. Keenan HA, Sun JK, Levine J et al (2010) Residual insulin production and pancreatic ss-cell turnover after 50 years of diabetes: Joslin Medalist Study. Diabetes 59:2846-2853

3. Sun JK, Keenan HA, Cavallerano JD et al (2011) Protection from retinopathy and other complications in patients with type 1 diabetes of extreme duration: the joslin 50-year medalist study. Diabetes Care 34:968-974

4. Salomon D, Meda P (1986) Heterogeneity and contact-dependent regulation of hormone secretion by individual B cells. Exp Cell Res 162:507-520

5. Pipeleers D, Kiekens R, Ling Z, Wilikens A, Schuit F (1994) Physiologic relevance of heterogeneity in the pancreatic beta-cell population. Diabetologia 37(Suppl 2):S57-S64

6. Pipeleers DG (1992) Heterogeneity in pancreatic beta-cell population. Diabetes 41:777-781

7. Talchai C, Xuan S, Lin HV, Sussel L, Accili D (2012) Pancreatic beta cell dedifferentiation as a mechanism of diabetic beta cell failure. Cell 150:1223-1234
8. Weir GC, Aguayo-Mazzucato C, Bonner-Weir S (2013) $\beta$-Cell dedifferentiation in diabetes is important, but what is it? Islets 5: 233-237

9. Dor Y, Brown J, Martinez OI, Melton DA (2004) Adult pancreatic beta-cells are formed by self-duplication rather than stem-cell differentiation. Nature 429:41-46

10. Smukler SR, Arntfield ME, Razavi R et al (2011) The adult mouse and human pancreas contain rare multipotent stem cells that express insulin. Cell Stem Cell 8:281-293

11. Al-Hasani K, Pfeifer A, Courtney M et al (2013) Adult duct-lining cells can reprogram into beta-like cells able to counter repeated cycles of toxin-induced diabetes. Dev Cell 26:86-100

12. Pfeifer A, Courtney M, Ben-Othman N et al (2013) Induction of multiple cycles of pancreatic beta-cell replacement. Cell Cycle 12: 3243-3244

13. Xu X, D'Hoker J, Stange G et al (2008) Beta cells can be generated from endogenous progenitors in injured adult mouse pancreas. Cell 132:197-207

14. Chera S, Baronnier D, Ghila L et al (2014) Diabetes recovery by age-dependent conversion of pancreatic delta-cells into insulin producers. Nature 514:503-507

15. Collombat $\mathrm{P}, \mathrm{Xu} X$, Ravassard $\mathrm{P}$ et al (2009) The ectopic expression of Pax 4 in the mouse pancreas converts progenitor cells into alpha and subsequently beta cells. Cell 138:449-462

16. Thorel F, Nepote V, Avril I et al (2010) Conversion of adult pancreatic alpha-cells to beta-cells after extreme beta-cell loss. Nature 464:1149-1154

17. Tamplin OJ, Kinzel D, Cox BJ, Bell CE, Rossant J, Lickert H (2008) Microarray analysis of Foxa2 mutant mouse embryos reveals novel gene expression and inductive roles for the gastrula organizer and its derivatives. BMC Genomics 9:511

18. Burtscher I, Lickert H (2009) Foxa2 regulates polarity and epithelialization in the endoderm germ layer of the mouse embryo. Development 136:1029-1038

19. Gegg M, Bottcher A, Burtscher I et al (2014) Flattop regulates basal body docking and positioning in mono- and multiciliated cells. eLife 3. doi:10.7554/eLife. 03842

20. Lange A, Gegg M, Burtscher I, Bengel D, Kremmer E, Lickert H (2012) Fltp ${ }^{\text {T2AiCre }}$ : a new knock-in mouse line for conditional gene targeting in distinct mono- and multiciliated tissues. Differ Res Biol Divers 83:S105-S113

21. Seifert JR, Mlodzik M (2007) Frizzled/PCP signalling: a conserved mechanism regulating cell polarity and directed motility. Nat Rev Genet 8:126-138

22. Wallingford JB (2012) Planar cell polarity and the developmental control of cell behavior in vertebrate embryos. Annu Rev Cell Dev Biol 28:627-653

23. Nusse R (2005) Wnt signaling in disease and in development. Cell Res 15:28-32

24. Clevers H (2006) Wnt/ $\beta$-catenin signaling in development and disease. Cell 127:469-480

25. Wang Y, Nathans J (2007) Tissue/planar cell polarity in vertebrates: new insights and new questions. Development 134:647-658

26. Rodriguez-Seguel E, Mah N, Naumann H et al (2013) Mutually exclusive signaling signatures define the hepatic and pancreatic progenitor cell lineage divergence. Genes Dev 27:1932-1946

27. Cortijo C, Gouzi M, Tissir F, Grapin-Botton A (2012) Planar cell polarity controls pancreatic beta cell differentiation and glucose homeostasis. Cell Rep 2:1593-1606

28. Blum B, Hrvatin SS, Schuetz C, Bonal C, Rezania A, Melton DA (2012) Functional beta-cell maturation is marked by an increased glucose threshold and by expression of urocortin 3. Nat Biotechnol 30:261-264

29. Bonner-Weir S (1988) Morphological evidence for pancreatic polarity of beta-cell within islets of Langerhans. Diabetes 37:616-621 
30. Katsuta H, Akashi T, Katsuta R et al (2010) Single pancreatic beta cells co-express multiple islet hormone genes in mice. Diabetologia 53:128-138

31. Heimberg H, De Vos A, Vandercammen A, Van Schaftingen E, Pipeleers D, Schuit F (1993) Heterogeneity in glucose sensitivity among pancreatic beta-cells is correlated to differences in glucose phosphorylation rather than glucose transport. EMBO J 12:28732879

32. Stefan Y, Meda P, Neufeld M, Orci L (1987) Stimulation of insulin secretion reveals heterogeneity of pancreatic B cells in vivo. J Clin Invest 80:175-183
33. Bader E, Migliorini A, Gegg M et al (2016) Identification of proliferative and mature $\beta$-cells in the islet of Langerhans. Nat Med. doi:10.1038/nature18624

34. Speier S, Nyqvist D, Cabrera O et al (2008) Noninvasive in vivo imaging of pancreatic islet cell biology. Nat Med 14:574-578

35. Kalwat MA, Thurmond DC (2013) Signaling mechanisms of glucose-induced F-actin remodeling in pancreatic islet beta cells. Exp Mol Med 45:e37 